\title{
Die Phytopharmazie ist eine sehr lebendige Wissenschaft
}

An der Zürcher Hochschule für Angewandte Wissenschaften (ZHAW) in Wädenswil fungiert Dr. Evelyn Wolfram als Nachfolgerin von Professor Dr. Beat Meier an der Spitze der Fachgruppe Phytopharmazie. Sie möchte neue Forschungsansätze verfolgen und Bewährtes bewahren.

\section{Seit fast einem Jahr sind}

Sie Leiterin der Fachgruppe

Phytopharmazie an der

ZHAW in Wädenswil, Frau Dr.

Wolfram. Wie fühlt sich das an?

Dr. Evelyn Wolfram: Sehr schön. Ich konnte schon viel erleben und gestalten. Bevor ich das Amt antrat, habe ich 5 Jahre bei Professor Beat Meier gearbeitet und war quasi bei ihm in der Lehre. Deshalb habe ich als seine Nachfolgerin optimal vorbereitet beginnen können. Ich hatte nicht gedacht, dass es so einen grossen Unterschied macht, wenn man selbst am Ruder ist. Die Bilanz ist äusserst positiv. Es gibt sehr viele Dinge, die von früher weitergeführt werden können. Dass Beat Meier, der auch noch bei uns im Team ist, mit seinem ganzen Wissen zur Verfügung steht, ist eine grosse Bereicherung. Gerade in unserer «Branche», in der es ja um traditionelles Wissen geht, das überliefert und weitergegeben wird, ist ein sehr harmonischer Generationenwechsel mit Bewahrung und Weitergabe des Wissens sehr wertvoll.

\section{Möchten Sie in der Forschung neue Akzente setzen oder primär das weiterführen, was bisher gemacht wurde?}

Ich möchte beides. Ein Standbein, das in der Vergangenheit sehr erfolg-

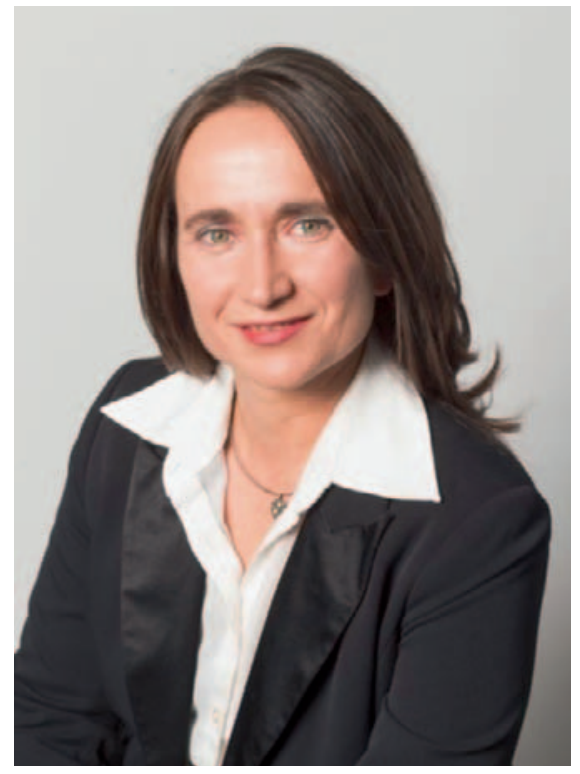

reich aufgebaut wurde, ist die phytoanalytische Forschung. In ihr arbeiten wir sehr viel sowohl für das schweizerische als auch für das europäische Arzneibuch. Wir engagieren uns in den Fachausschüssen für Phytochemie und für Komplementärmedizinische Arzneimittel beim Erstellen und Revidieren zahlreicher Monographien und erhalten Aufträge von der Schweizer Pharmakopöeorganisation, die bei Swissmedic angesiedelt ist.

Das Institut ist durch Beat Meier in diesem Gremium auch in den nächsten Jahren noch vertreten, und wenn er dann wirklich ganz in den wohlverdienten Ruhestand auch bei diesen Ämtern gehen möchte, haben wir mit unserem erfahrenen analytischen Chemiker Samuel Peter bereits ein sehr kompetentes designiertes Ausschussmitglied. Wir sind nach wie vor ein sehr wichtiger Ansprechpartner für die phytopharmazeutische Industrie in der Schweiz und in Europa. Weil wir in der Schweizer Szene sehr gut vernetzt sind und dieses Phytonetzwerk auch koordinieren, sehen wir nach wie vor unsere Aufgabe darin, Ansprechpartner für die Industrie zu sein, und ich möchte das weiter ausbauen.

\section{Sie haben keine Berührungsängste zwischen Akademie und Industrie? Das ist ja beispielsweise in der klassischen Pharmaforschung oder der Biotechnologie oft ein heikler Punkt.}

Die Fachhochschulen sind prädestiniert für diese Aufgabe und können genau in diesem Spannungsfeld funktionieren. Wir machen angewandte Forschung, denn die Industrie braucht Ansprechpartner, die auf ihre konkreten Probleme eingehen können. Die Sicht von aussen, die wir bieten, ist wichtig, denn man wird irgendwann betriebsblind, und in der Industrie ist neben dem Tagesgeschäft für Vieles keine Zeit mehr vorhanden. Hier springen wir mit unserem Know-how ein. Man darf auch nicht vergessen, 
dass die Fachhochschulen für den Nachwuchs und für die Fachkräfte sorgen, die die Industrie braucht. Bei der Bearbeitung von Aufgaben aus der Industrie bilden wir gleichzeitig Studierende an deren praktischen, täglichen Herausforderungen aus. Ausserdem beschreiben wir «nur» und machen keine Wertung, wie man es in der klinischen Forschung erwartet, wo man möchte, dass das zu untersuchende Präparat mindestens gleich gut und am liebsten sogar besser als das herkömmliche Präparat wirkt.

\section{Diese Studierenden können dann wieder in die Phytoindustrie gehen?}

Das ist der Rückkopplungseffekt der Zusammenarbeit: Die Industrie unterstützt uns, wir bilden für sie sehr wertvolle Fachleute aus - und dadurch gibt es einen Austausch. Es ist sehr gut, dass eine Firma die Aussensicht auf ihre Produkte und auf ihre Methoden zulässt und sich helfen lässt. Es ist in anderen Branchen auch üblich, externe Berater hinzuzuziehen.

Die Aufgaben in der Phytoindustrie sind so vielfältig, dass es viel Kompetenz in ganz verschiedenen Bereichen braucht. Da ist eine Fachhochschule wie die ZHAW sehr gut geeignet, dieses Wissen anzubieten. Wenn wir beispielsweise über ein Spezialwissen in der Fachgruppe Phytopharmazie nicht verfügen, ist es sehr wahrscheinlich in einer anderen Arbeitsgruppe oder einem anderen Institut im Department of Life Sciences and Facility Management der ZHAW vorhanden. Dann wird ein Konsortium innerhalb der Hochschule oder innerhalb des Phytonetzwerks zusammengestellt. Wir wollen dafür sorgen, dass die Kunden die Kompetenzen bekommen, die sie benötigen - unabhängig davon, welches Institut sie bereitstellt.

\section{Das macht den \\ Servicecharakter der ZHAW \\ für die Phytopharmaindustrie in der Schweiz aus?}

Ja. Als wie wichtig das von der Phytoindustrie eingeschätzt wird, zeigt auch die Unterstützung, die wir durch die Wirtschaft erfahren. Einige Firmen haben sich zusammengeschlossen, um uns finanziell mit einer Grundfinanzierung zu unterstützen, damit unsere Fachgruppe überhaupt bestehen und unter Sicherstellung der akademischen Unabhängigkeit das so entstandene Phytonetzwerk mit wissenschaftlicher Expertise unterstützen und koordinieren kann. Unter Umständen würde ein Institut für Biotechnologie gar nicht auf die Idee kommen, eine phytopharmazeutische Gruppe alleine zu betreiben. Dann wäre die Konzentration auf dieses Fachgebiet nicht gegeben, und es fehlten die Spezialisten, die sich mit den sehr komplexen Mischungen auskennen und die vonnöten sind, weil Phytopharmaka auch analytisch eine Herausforderung sind.

\section{Wenn Sie auf die letzten 10 Jahre zurückschauen, was waren die grössten Erfolge der Phytopharmazie und wo hat sich am meisten etwas getan?}

Lassen Sie mich da etwas länger zurückblicken. In den 1950er- bis 1960er-Jahren ist auch in der klassischen Pharmaindustrie sehr viel Naturstoffforschung betrieben worden. Die Firmen haben Pflanzen gesammelt, Extrakte gemacht und sie in ihre Wirkstoffsammlungen aufgenommen - oft mit dem Ziel, Einzelwirkstoffe zu isolieren. In den 1980er-Jahren und später hat man gedacht: «Ach, das ist doch ein so zufälliges Vorgehen; das muss man systematisch angehen.» Dann hat man sich der kombinatorischen Chemie bedient und versucht, nach einer bestimmten systematischen Vorgehensweise Moleküle zu- sammenzubauen. Dabei ist man von den Extrakten weggekommen und hat sich nahezu ausschliesslich auf das Konzept «1 Molecule - 1 Target» konzentriert. Die Phytoforschung hat diesen Weg nicht beschritten und ist ihrer Tradition treu geblieben. Das Multikomponentengemisch aus einer oder mehreren Heilpflanzen wird als Wirkstoff betrachtet. Dieser Ansatz, die Vielfalt der Wirkstoffe einer Pflanze $\mathrm{zu}$ nutzen, wird von den Patienten nach wie vor geschätzt.

Aus den Erfolgsgeschichten der letzten Jahrzehnte kann man sicherlich die des Johanniskrauts bei der Behandlung von Depressionen hervorheben. Wissenschaftlich wurde festgestellt, dass Johanniskrautextrakte gleich gut wirken wie einzelne Substanzen, die synthetisch hergestellt wurden. Unter bestimmten Umständen ist Johanniskraut auch das Mittel der Wahl, weil es bei bestimmten Patientinnen und Patienten weniger unangenehme Nebenwirkungen hat. Einzig in Kombination mit Sonnenbestrahlung oder hormonellen Verhütungsmitteln gibt es Anwendungseinschränkungen. Der grosse Durchbruch für das Johanniskraut war der Cochrane-Review, der die Daten zusammengefasst und klar gezeigt hat: Die Wirkung von Johanniskraut lässt sich nachweisen - es ist also ein evidenzbasiertes Medikament.

\section{Wie beurteilen Sie die Innovationsgeschwindigkeit der Phytoindustrie?}

Diese existiert im eigentlichen Sinne gar nicht. Das Haupthindernis für Innovationen ist, dass die gesetzlichen Anforderungen für eine Zulassung so hoch sind, dass sie von der Phytoindustrie für neue oder weniger bekannte Arzneipflanzen und für neue Indikationen bereits verwendeter Heilpflanzen so gut wie nicht finanzierbar sind. Die Phytotherapeutika sind vergleichsweise günstige Präparate, und so besteht ein Ungleichgewicht zwi- 
schen notwendigen Investitionen in teure klinische Studien und dem Marktpotenzial, das auch von der Offenheit der behandelnden Ärztinnen und Ärzte gegenüber Phytotherapeutika abhängt.

\section{Wenn man jedoch das Vertrauen der Patienten in diese Medikamente anschaut, erscheint dieses als ungebrochen und gross.}

Ganz genau, doch ich habe das Gefühl, dass bei den Patienten sehr viele Konzepte der Komplementärmedizin komplett vermischt und etwa die Homöopathie und die Phytotherapie im fast gleichen Atemzug genannt werden. Erstaunlich ist auch das Gefühl der Unbedenklichkeit der Naturheilverfahren, das sich bei den Menschen verbreitet beobachten lässt. Das hat vielleicht mit der erneuten Hinwendung zur Natur zu tun, die ein Trend ist. Ich denke, dass es wichtig ist, dass Patienten die Verfahren der Naturheilkunde nur begleitet durch eine Fachperson, sei es ein/e in Phytotherapie/Naturheilkunde ausgebildete/r Arzt/Ärztin oder Apotheker/in oder auch ein/e naturheilkundige/r Therapeut/Therapeutin, anwenden. Auf diese Weise sind auch die Zielführung und der Erfolg der Therapie wahrscheinlicher, wenn hingegen bei einer Selbstmedikation zu viel, zu kurz oder wild durcheinander irgendetwas angewendet wird.

\section{Sehr viele Schulmediziner wenden komplementär- medizinische Methoden an, wenn sie mit ihrem Latein am Ende sind?}

Ja, das ist möglich, wobei man aber immer wieder Ärztinnen und Ärzten sowie Apothekerinnen und Apothekern begegnet, die sehr aufgeschlossen sind. Das sind Medizinalpersonen, die sich beispielsweise von der Schweize-

Dr. Evelyn Wolfram promovierte im Fachgebiet Biotechnologie am Forschungszentrum Jülich, Deutschland, in Kooperation mit der TU München, Deutschland. Bei der Cytos Biotechnology AG in Schlieren bei Zürich sammelte sie wertvolle Erfahrungen in der Entwicklung und dem Qualitätsmanagement von pharmazeutischen Wirkstoffen und der Guten Herstellungspraxis (Good Manufacturing Practice (GMP)). Mit der Übernahme der Leitung der Verfahrensentwicklung Pharma des Pflanzenextraktherstellers Emil Flachsmann AG (heute Frutarom Switzerland Ltd.), Wädenswil, Schweiz, begann für sie ihr beruflicher Weg in der Arzneipflanzenforschung. Nach einem Abstecher in die Welt der Entwicklung und Qualitätssicherung kosmetischer Pflanzenextrakte bei Cosmetochem International Ltd., Steinhausen, Schweiz, ist Frau Dr. Wolfram seit 2009 für die Fachgruppe Phytopharmazie am Institut für Biotechnologie der Zürcher Hochschule für angewandte Wissenschaften (ZHAW) in Wädenswil im Bereich der analytischen und biotechnologischen Forschung und Entwicklung von Medizinalpflanzen und deren Produkten sowie der Lehre im Bachelorstudiengang Biotechnologie und dem Master-Studium Life Sciences tätig. Seit September 2014 hat Frau Dr. Wolfram als Dozentin die Leitung der Fachgruppe von Prof. Dr. Beat Meier übernommen.

Die ZHAW ist eine der grössten und renommiertesten Fachhochschulen der Schweiz. Das Departement Life Sciences und Facility Management in Wädenswil ist eines der anerkannten Kompetenzzentren in der Schweiz zu den Themen Ernährung, Gesundheit, Gesellschaft und Umwelt.

rischen Gesellschaft für Phytotherapie (SMGP) ausbilden lassen. Dabei spielt der Ausbildungszyklus der SMGP, der unter anderem an der ZHAW stattfindet, eine wichtige Rolle. Es besteht aber noch mehr Potenzial unter den medizinischen Fachpersonen, und ich hoffe, dass es in Zukunft eine noch grössere Aufgeschlossenheit gibt und sich mehr Therapeuten und Pharmazeuten entsprechend öffnen und ausbilden lassen. In Japan scheinen die traditionelle japanische Medizin und die Schulmedizin stark Hand in Hand $\mathrm{zu}$ arbeiten, aber dort werden alle $\mathrm{Me}-$ diziner auch in der traditionellen Medizin ausgebildet. Bei uns ist es schade, dass in der Ausbildung der jungen Ärzte die komplementärmedizinischen Verfahren fehlen und dass die unglaubliche Innovation und Technisierung der Medizin, die wir im Moment erleben, anderem wenig Raum lässt. Ärztinnen und Ärzte müssen heute mehr Dinge lernen als früher, und dann verzichtet man eben auf das, was sich zwar aus der Tradition heraus bewährt hat, aber wissenschaftlich nicht so gut erklärbar ist oder nicht der derzeitigen modernen Sichtweise entspricht.

\section{Könnte die Idee der modernen Biotechnologie, sich in der Natur auf die Suche nach möglichen Wirkstoffen zu machen und sie dann im Labor nachzubauen, auch der Phytopharmazie Auftrieb geben?}

Global ist die Forschung sehr aktiv, vor allem in China und anderen Ländern Asiens. In China möchte man die Traditionelle Chinesische Medizin auf einen naturwissenschaftlichen Boden stellen, und deshalb forscht man dort auch stark an der Aufklärung von Wirkmechanismen. Dabei interessiert man sich auch sehr für die molekulare Ebene. Auch in anderen Ländern, wie etwa in der Schweiz, haben in den letzten Jahren sehr viele Forschungsaktivitäten eingesetzt, die den molekularen Mechanismen auf der Spur sind. Dann ist es natürlich nur ein kleiner Schritt, bis man den Wirkstoff optimieren und vielleicht biotechnologisch herstellen kann. Das ist aber das Gebiet der klassischen Biotechnologie und nicht mein Ansatz.

\section{Weil es Ihnen um das «Gemisch» geht?}

Genau. Trennen und Isolieren ist Teil der Naturstoffforschung, aber für 
mich nur ein Werkzeug, um mehr über den Wirkstoff zu erfahren und auf dem Hintergrund der evidenzbasierten Medizin das Wirkprinzip besser zu verstehen. Die Biotechnologie wird in der Phytopharmazie an ganz anderer Stelle gebraucht. Im Hinblick auf in Zukunft immer knapper werdende Anbauflächen und die Notwendigkeit, Wildbestände aus Gründen der Nachhaltigkeit mehr zu schützen, stellt sich die Frage, wie der aus unserer Sicht hoffentlich steigende Bedarf an Heilpflanzen auch in Zukunft gedeckt werden kann. Es lohnt sich daher darüber nachzudenken, wie man von Anbauflächen und intensiver, schädlicher Sammelaktivität in der Wildnis unabhängiger werden könnte und die Versorgung mit den Rohmaterialien dennoch und vielleicht sogar ganzjährig sicherstellen kann. Im Moment gibt es bereits Probleme: Sehr viele Heilpflanzen können gar nicht angebaut werden, und man muss sie in der Natur suchen, wo sie teilweise rar werden, z.B. Arnica montana.

\section{Da wäre die biotechnologische Herstellung von Inhaltsstoffen eine valable Alternative?}

In erster Linie geht es um die Herstellung des natürlichen und keinesfalls genveränderten Pflanzenmaterials, das möglichst die gleichen Inhaltsstoffe enthält wie die wild gewachsenen oder angebauten Pflanzen. Das ist ein Forschungsthema, das mich an der ZHAW mehr und mehr beschäftigt und bei dem ich einen Schwerpunkt setzen möchte. Vergessen Sie nicht, dass mein beruflicher Hintergrund die Biotechnologie ist. Es ist möglich, Heilpflanzen aus der Wildnis im Labor zu kultivieren und diese wilden, inhaltsstoffreichen Sorten zu vermehren sowie auf Dauer und effizient zu sichern. So ist es auch möglich, das einzigartige natürliche Erbgut der inhaltsstoffreichen Ursprungspflanzen für nachfolgende Generationen zu bewahren. Angesichts der Gefährdung der natürlichen Vielfalt scheint es mir für die Phytopharmazie wichtig, sich mit diesen Zukunftsthemen auseinanderzusetzen.

\section{Weil man so die Rohstoffe ökologischer und nachhaltiger bekommen kann?}

Ja, denn die Frage, woher man die Rohstoffe bekommt, um Phytotherapeutika herzustellen, wird in der Diskussion um die Wirkstoffe aus der Natur häufig ausgeblendet. Eine Phytopharmafirma hat mehrere Möglichkeiten, Rohstoffe für die Produktion zu bekommen. Sie kann versuchen, Anbau zu betreiben, wenn das geht und wenn die Pflanze das erlaubt. Dazu muss die Firma Bauern finden, die das umsetzen. Wenn man sehr viel Ware benötigt und nicht nur einmal im Jahr produzieren will, ist man auf den Weltmarkt angewiesen oder hat weltweit Bauern unter Vertrag, die sich auch auf der südlichen und der nördlichen Hemisphäre befinden, um ganzjährig produzieren zu können. Die mangelnde zeitliche Verfügbarkeit der Rohstoffe ist eine grosse Limitierung, wenn ein Produkt in grossem Massstab verkauft wird. Wenn man von solchen Einschränkungen unabhängig wäre, also von Anbauzeiten oder auch von Ackerflächen, sondern in Gewächshäusern kultivieren könnte, wäre das ein grosser Fortschritt. Allerdings rufen solche Ideen Kritiker auf dem Plan, die an die Kraft der Natur glauben und sagen: «Die Pflanzen wachsen ohne richtiges Sonnenlicht, der Wind fehlt, der Boden ist nicht natürlich und die Bodenflora fehlt.» Trotzdem ergeben solche Anbaumethoden Sinn, weil man so neben dem Nachschub auch die Qualität der Rohstoffe und den Wirkstoffgehalt kontrollieren kann.

\section{Die Nachhaltigkeit der Rohstoffe wird komplett ausgeblendet.}

Tatsächlich erscheint in den Medien kaum etwas zu dem Thema, jedoch misst ihm die Branche trotzdem eine grosse Bedeutung bei. Es gibt einige Firmen, die auf die Nachhaltigkeit bei der Gewinnung ihrer Rohstoffe besonders achten. Das führt zum Teil dazu, dass sie sich beim Marketing zurückhalten, weil sie grössere Mengen gar nicht mehr liefern können, ohne ihre eigenen Regeln zu verletzen.

\section{Für die Phytopharmazie sind also verfahrenstechnische Methoden eine riesige Chance, um die Lieferengpässe zu beseitigen und die Nachhaltigkeit der Produktion sicherzustellen?}

Ja, aber es gibt ein paar Probleme wie das der Gleichwertigkeit. Eine Pflanze aus der biotechnologischen Kultur hat unter Umständen nicht die gleichen Inhaltsstoffe wie eine aus der Natur. In der Kultur haben es die Pflanzen gemütlich - ihnen geht es gut, sie haben keinen Stress und müssen bestimmte Inhaltsstoffe zum Zweck der Abwehr oder Verteidigung nicht produzieren. Vor einigen Jahrzehnten hatte man bei Echinacea grosse Hoffnung auf die Pflanzenkultur gesetzt und versuchte, sie in Zellkultur zu ziehen. Das hat aber nicht den gewünschten Effekt gezeigt, meist war der Wirkstoffgehalt der erzeugten Biomasse zu gering, und man hat gemerkt, dass sich das Verfahren nicht lohnt.

Es ist sehr viel Forschung erforderlich, und die Methoden stecken noch in den Kinderschuhen. Wenn ich mir anschaue, was an Publikationen eingereicht wird, habe ich den Eindruck, dass das Thema doch wieder im Kommen ist. Der Druck nimmt zu. Man schaut in die Zukunft und sieht, dass Wasser weniger wird, die Bevölkerung wächst und die Flächen knapper wer- 
den. Man möchte die Urwälder behalten und die Schutzzonen durchsetzen, was bei der Wildsammlung mit vielen Menschen, die durch den Wald gehen und die Heilpflanzen ausrupfen, kaum möglich ist. Wenn die Phytopharmazie weiter wachsen soll, stossen wir irgendwann an die Grenzen.

\section{Sie kooperieren an der ZHAW mit Forschern aus Rumänien und Bulgarien?}

Das sind sehr interessante Kontakte, die ich in Zukunft noch weiter ausbauen möchte. Die Länder Osteuropas sind Lieferanten der Rohstoffe für viele Phytotherapeutika. Dort wird auch entsprechend viel Forschung betrieben. Vor allem Polen, Rumänien, Bulgarien oder die Türkei exportieren grosse Mengen an Heilpflanzen. Nach dem Fall des Eisernen Vorhangs war es notwendig, sich dort wirtschaftlich neu $\mathrm{zu}$ orientieren. Dort wurden Händlersysteme aufgebaut, weil die Nachfrage aus dem Westen plötzlich da war. Man spürt, dass hinter der Phytopharmazie eine grosse Industrie steckt. Die Firmen haben sehr unterschiedliche Philosophien, wie sie mit Schwierigkeiten umgehen, die diese industriellen Massstäbe mit sich bringen. In der Schweiz achtet man sehr darauf, wo die Rohstoffe herkommen. Es gibt einige Firmen, die sich nur mit Rohstoffbeschaffung befassen und in bestimmten Ländern Bauern unter Vertrag nehmen. Man weiss dann ganz genau, von welchem Feld eine Heilpflanze kommt.

\section{Im Moment ist die Synthetische Biologie en vogue, d.h., die Idee, dass man Wirkstoffe analysiert, deren genetische Kodierung nachbaut und sie dann biotechnologisch erzeugt.}

Das sind sehr interessante Verfahren, die nicht in unser Forschungsgebiet der Arbeitsgruppe fallen. An der ZHAW haben wir in anderen Fachgruppen aber die entsprechenden Möglichkeiten dazu. Das ist auch einer der Vorteile, die wir in Wädenswil haben: Wir haben im Department of Life Sciences und Facility Management sehr viele unterschiedliche Kompetenzen und können in Netzwerken spannende Fragestellungen bearbeiten. Eine Nachbargruppe um Prof. Rainer Riedl beschäftigt sich z.B. mit der medizinischen Chemie, die von der Molekülstruktur eines Naturstoffs ausgeht und dann den Wirkstoff aus pharmazeutischen Gründen, z.B. Bioverfügbarkeit oder Steigerung der Wirksamkeit am Wirkort, verändert. Mittels Computersimulation kann ermittelt werden, mit welcher Wahrscheinlichkeit der Wirkstoff an welche Rezeptoren andocken kann. Und auf einmal passt es ganz wunderbar und es ist ein neuer Wirkstoff, der der Natur eben nur entlehnt, aber nicht genau gleich ist. Der Wirkstoff des Präparats Aspirin, abstammend von der Salicylsäure, ist da ein bekanntes Beispiel.

\section{Aber das ist absolut fern dessen, was wir unter komplementärmedizinischer Phytopharmazie verstehen.}

Das stimmt schon, und deshalb ist es nicht unser Gebiet. Aber betrachten Sie die Natur bitte nicht als statisch. Die Wirkstoffe werden ja bereits im Mund und dann in unserem Verdau- ungssystem individuell verändert, weil sich das Mikrobiom im Darm von Mensch zu Mensch unterscheidet. Jeder Mensch hat seine eigene Flora und die hat einen Einfluss auf die Verfügbarkeit im Körper und möglicherweise auch die Wirksamkeit. Es gibt da spannende Ansätze, die in die Richtung der personalisierten Medizin gehen. Ich wurde angefragt, mich an einem ZHAW-Symposium über personalisierte Medizin zu beteiligen. Da habe ich mich das erste Mal gefragt, was die Phytopharmazie mit dem derzeit viel diskutierten Begriff der personalisierten Medizin zu tun hat. Beim näheren Hinsehen kann man die Phytotherapie und Komplementärmedizin im Kern als eine Form der personalisierten Medizin ansehen. Rezepturen werden auf den Patienten und oft nicht nur bezogen auf die konkreten Beschwerden, sondern auch auf den Allgemeinzustand und die Lebensumstände zugeschnitten: Sie werden vom Therapeuten aus der Vielfalt der Phytotherapeutika und ergänzend mithilfe von beispielsweise Magistralrezepturen dem Patienten so verschrieben, dass sie genau auf seine Symptomatik oder Multimorbidität passen. Es gibt auch entsprechende Publikationen aus der Tibetischen Medizin, die in diese Richtung weisen. Ich glaube, an dieser Entwicklung sieht man sehr deutlich, dass die Phytopharmazie eine sehr lebendige Wissenschaft ist, die ständig Neues hervorbringt und sehr spannende Ansätze entdeckt, obwohl sie ihrem traditionellen Ansatz treu bleibt. Dass sie bewährte Multikomponenten-Wirkstoffe, moderne Analytik und die neuen Methoden der Biologie zusammenbringt, gehört zu den Dingen, die mich für sie begeistert, und mein Team und ich werden uns auf dem Gebiet weiterhin mit grossem Engagement im Sinne der Phytopharmazie auf vielfältige Weise einbringen.

Interview: Oliver Klaffke 